\title{
Large pulmonary hamartoma with "popcorn" like calcification
}

\author{
K. Madan1, S. Sharma1, N. Singh1, S. Radhika²
}

A 17 year old boy presented with a history of ill-defined right sided chest discomfort for three years and dry cough of five months duration. There was no history of hemoptysis, wheezing, dyspnea or constitutional symptoms. The patient had been treated with standard 6 month four drug anti-tubercular treatment (ATT) twice during the last two years by two separate physicians on the basis of an 'abnormal' chest radiograph, without any improvement in symptoms. However, he had not experienced any improvement in his symptoms during either of these two courses of ATT. He also categorically denied any history of sputum production or having had sputum examination prior to initiation of ATT. General physical examination was unremarkable and vital signs were normal. Chest examination revealed reduced intensity of breath sounds on the right side.

A posteroanterior chest radiograph (figure 1) showed a large calcified mass lesion arising from the right hilar region along with tenting of right hemidiaphragm and obscuration of right costophrenic angle. The pattern of calcification resembled a large 'popcorn'. CT scan of the thorax demonstrated a solitary, large $(8.2 \times 8 \times$ $7.8 \mathrm{~cm}$ ) densely calcified mass lesion (figure 2) in right lower lobe. The margins of the mass did not show spiculation. There was no mediastinal or hilar lymphadenopathy. There were no nodules or any other parenchymal abnormalities in the right upper lobe or the left lung. In view of characteristic radiological features, a diagnosis of pulmonary hamartoma was considered. In view of an atypical presentation and symptomatic disease, CT guided fine needle aspiration cytology (FNAC) from the mass (figure 3) was performed which showed predominantly chondromyxoid matrix without any bronchial epithelium. There was no necrosis and no evidence of any granulomatous inflammation. Stain for acid fast bacilli was negative. The diagnosis of a benign pulmonary hamartoma was confirmed. In view of the presence of symp- toms attributable to the large chondroid hamartoma, the patient was counselled regarding surgical removal of the tumour and referred to the thoracic surgical unit of our institute. However, the patient did not get the surgical resection carried out.

The radiological manifestations of certain disorders have been associated with certain kinds of food in view their striking resemblance with the latter. The recognition of these classical food signs can help in narrowing down the differential diagnosis or in some cases establish a confident diagnosis on radiology alone [1]. The presence of popcorn calcification in a pulmonary hamartoma is one such characteristic radiologic "food sign". The typical radiological pattern of popcorn calcification (seen in 10$15 \%$ of pulmonary hamartomas on chest radiographs) when present, is highly suggestive of the diagnosis [2].

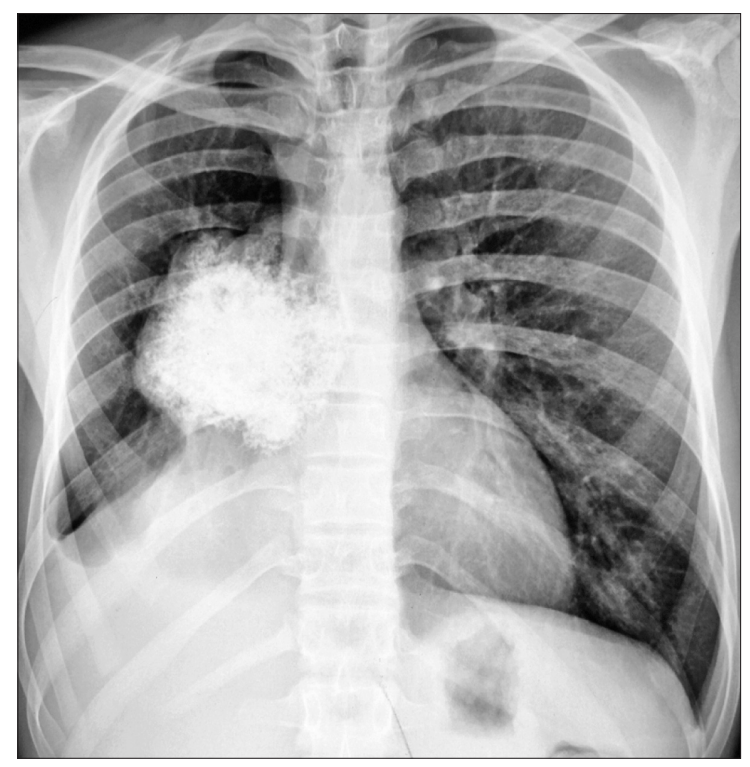

Fig. 1. - Chest radiograph showing a large mass lesion arising from the right hilar region with 'popcorn' calcification along with tenting of right hemidiaphragm and obscuration of right costophrenic angle.
Monaldi Arch Chest Dis 2011; 75: 4, 243-244

\section{Keywords: \\ Hamartoma, \\ Calcification,}

Mass.

${ }^{1}$ Department

of Pulmonary Medicine,

${ }^{2}$ Department of Cytology and Gynaecological

Pathology,

Post Graduate Institute

of Medical Education

and Research (PGIMER),

Chandigarh, India.

Correspondence: Karan Madan MD Senior Resident

Department

of Pulmonary Medicine

Post Graduate Institute

of Medical Education and Research (PGIMER)

Sector 12, Chandigarh,

India 160012; e-mail:

drkaranmadan@gmail.com 

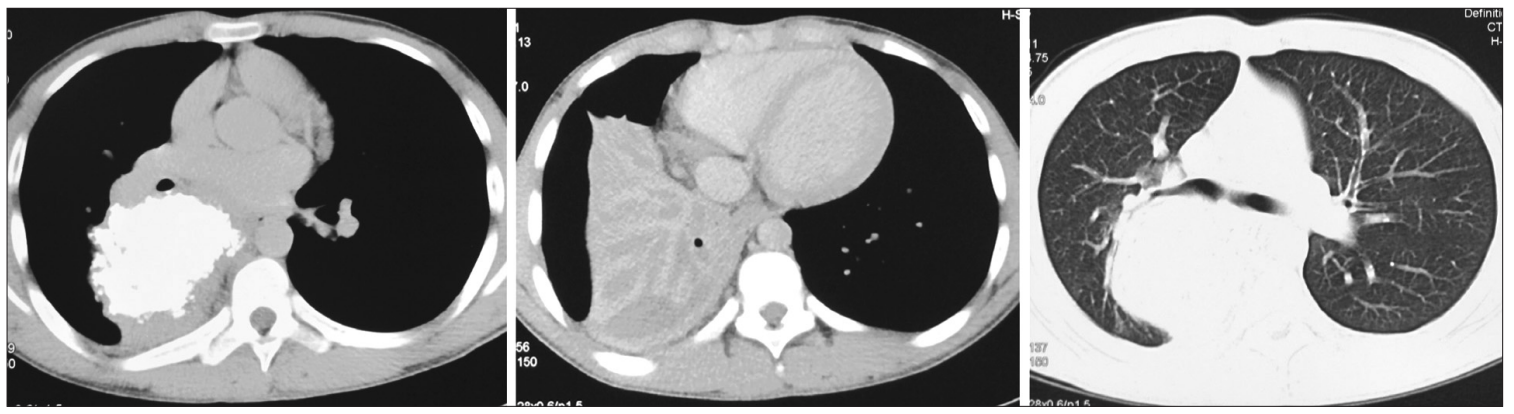

Fig. 2. - Computed tomography scan of the thorax showing a solitary $8.2 \times 8$ $x 7.8 \mathrm{~cm}$ densely calcified mass lesion (left panel) in right lower lobe along with collapse-consolidation of right middle lobe (middle panel). There were no parenchymal abnormalities in the right upper lobe or the left lung (Right panel).

Pulmonary hamartomas, accounting for approximately $6 \%$ of all solitary pulmonary nodules are the most common benign lung tumours and are usually asymptomatic. They can rarely cause symptoms due to large size. Most are parenchymal but uncommonly can be present in an endobronchial location. They are more common in males with peak incidence in the sixth or seventh decade of life [3]. FNAC is a useful diagnostic modality which by providing a rapid and accurate diagnosis in most of the cases, can avert the need for a diagnostic thoracotomy [2]. On pathological examination, most demonstrate a predominant chondroid differentiation $(80 \%)$ as was seen in the index case [4].

Asymptomatic hamartomas usually require no further treatment. The indications of removal include a rapid growth of tumour with symptoms, suspected primary or secondary malignant tumour or endobronchial location of lesion with post obstructive complications. Surgical removal is the curative modality in such instances. In patients with large tumors who do not choose to undergo resection, a lifelong follow up may be advisable.

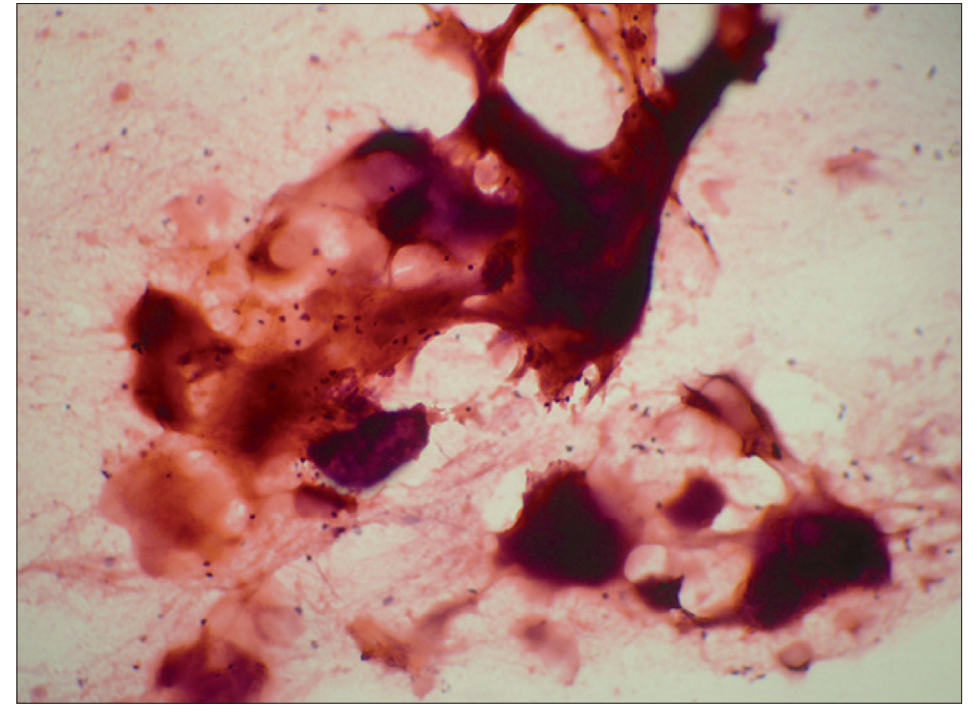

Fig. 3. - Microphotograph of fine needle aspiration cytology smear from solitary pulmonary lesion showing chondromyxoid material with few scattered epithelial and inflammatory cells in the background but without any necrosis or granulomatous inflammation (Hematoxylin and eosin; 200X). 